\title{
ARTICLE OPEN \\ Reversible conductivity recovery of highly sensitive flexible devices by water vapor
}

\author{
Yuting Wang ${ }^{1,2}$, Yingchun $\mathrm{Su}^{2,3}$, Zegao Wang ${ }^{2}$, Zhongyang Zhang ${ }^{2}$, Xiaojun $\mathrm{Han}^{3}$, Mingdong Dong ${ }^{2}$, Lifeng Cui ${ }^{1}$ and Menglin Chen ${ }^{2}$
}

With decreasing size of integrated circuits in wearable electronic devices, the circuit is more susceptible to aging or fracture problem, subsequently decreasing the transmission efficiency of electricity. Micro-healing represents a good approach to solve this problem. Herein, we report a water vapor method to repair microfiber-based electrodes by precise positioning and rapid healing at their original fracture sites. To realize this micro-level conducting healing, we utilize a bimaterial composed of polymeric microfibers as healing agents and electrically conductive species on its surface. This composite electrode shows a high-performance conductivity, great transparency, and ultra-flexibility. The transmittance of our electrode could reach up to 88 and $90 \%$ with a sheet resistance of 1 and $2.8 \Omega \mathrm{sq}^{-1}$, respectively, which might be the best performance among Au-based materials as we know. Moreover, after tensile failure, water vapor is introduced to mediate heat transfer for the healing process, and within seconds the network electrode could be healed along with recovering of its resistance. The recovering process could be attributed to the combination of adhesion force and capillary force at this bimaterial interface. Finally, this functional network is fabricated as a wearable pressure/ strain sensing device. It shows excellent stretchability and mechanical durability upon 1000 cycles.

npj Flexible Electronics (2018)2:31; doi:10.1038/s41528-018-0043-z

\section{INTRODUCTION}

Flexible conductive materials and transparent electrodes with high conductivity, stretchability, healability, and integrable characteristics are indispensable in next generation wearable electronics. $^{1-4}$ Especially, conductive materials with healable characteristic could reduce the replacement and maintenance costs while improving the reliability and lifetime of the devices. For electronic devices, aging or mechanical damage of electrical components may ruin the entire circuit or cause an irreversible crush. Such failures could be completely avoided by healable conductive materials. ${ }^{5-8}$ More recently, miniaturization of integrated circuits became a new trend in wearable electronic devices. Subsequently, the circuit is more susceptible to suffer aging or fracture problem because of the decreasing size. ${ }^{9}$ Micro-scale healing seems to be crucial for the integrated circuits to regain the transmission efficiency of devices. Restructuring is an important concept to build a large device system which consists of different components. ${ }^{10,11}$ Furthermore, micro-healing contributes a new method to reconstruct work packages and create a 3D configuration. ${ }^{12}$ Another potential application for these healable materials lies in electronic artificial skins and bioelectronics, ${ }^{1,13}$ inspired from healability as one of the key functions for human skin. ${ }^{14-17}$ Given that skin is the largest organ of the human body, it provides rich biometric interface signals such as body temperature, blood pressure, heart beats, and glucose level, etc. For example, Xiao et al. demonstrated an advanced e-skin with rehealable, fully recyclable, and malleable properties that can sense pressure, flow, temperature, and humidity. ${ }^{8}$ Therefore, bioinspired electronic skin (e-skins) having comparable functionalities and mechanical properties as natural skin has been of great interest. ${ }^{16}$
Recently, metal-based fibers have attracted great attention on transparent electrodes, strain/pressure sensors, and heaters, because of their great potential for invisible integrated circuits and wearable sensing devices for e-skins etc. ${ }^{19-24}$ However, highly conductive materials such as metal-based materials normally require ultra-high temperature or high-energy laser to reconstruct electrostatic interactions. ${ }^{25,26}$ Therefore, capsule-based methods have been developed for fabrication of healable conducting materials, which use polymeric microcapsules as the healing agents and incorporated with conductive species. ${ }^{27-31}$ Intriguingly, liquid metals such as eutectic gallium indium (EGaln) allows facile healing and reconstruction at ambient temperature, but their intrinsic liquid state demands dedicate confinement for application in integrated circuits and transparent devices. ${ }^{32}$ It is reported that the adhesive force between interdiffusion chain segments in polymeric agents is mainly driven by van der Waals interactions, and this force was found to increase along with the increasing temperature. ${ }^{33-36}$ Especially, at glass transition temperature, there will be highly mobile polymer chains, which will facilitate the healing process. ${ }^{37}$ Although most of healable materials can recover the structure and functions from damage, in situ micro-scale healing on its fractured interfaces for transparent electrodes has not been investigated. Comparing with severe damage such as cuts and cracks, stretching damage is omnipresent for flexible and stretchable sensor devices during daily use. Thus, precise micro-scale positioning and rapid healing is crucial for wearable electronics in practice.

For a couple decades, solution electrospinning provides a facile and versatile way to fabricate various functional fibers. ${ }^{24,38}$ However, it is hard to control the pattern or the network

\footnotetext{
${ }^{1}$ School of Environment and Civil Engineering, Dongguan University of Technology, 523808 Guangdong, China; ${ }^{2}$ Department of Engineering and Interdisciplinary Nanoscience Center, Aarhus University, DK-8000 Aarhus C, Denmark and ${ }^{3}$ State Key Laboratory of Urban Water Resource and Environment, School of Chemistry and Chemical Engineering, Harbin Institute of Technology, 92 West Da-Zhi Street, 150001 Harbin, China

Correspondence: Lifeng Cui (Icui@dgut.edu.cn) or Menglin Chen (menglin@eng.au.dk)
}

Received: 28 June 2018 Accepted: 8 November 2018

Published online: 21 December 2018 
architecture through solution electrospinning, which hinders its application on integrated devices. More recently, melt electrospinning writing (MEW), a combination of melt electrospinning and 3D-printing enables design and fabricate microfibers with controllable architectures. Charged polymer melts with less bending instability allow facile fabrication different desirable patterns, which holds great potential in integrated circuits and advanced wearable electronics. Further, the use of polymer fibers as templates for sputtering metal on the surface facilitates to produce high-quality transparent electrodes. ${ }^{39,40}$

Herein, we proposed a facile water vapor method to achieve a microscopic level of conductive healing. We utilize a bimaterial composed of polymeric microfibers as healing agents and electrically conductive species on the surface. After tensile failure under different strains, this bimaterial could be quickly healed along with recovery of its conductivity within seconds. Even after cutting into two pieces, this electrode can be quickly assembled, where both mechanical strength and conductivity can be recovered. This performance also exhibits good stability and repeatability in cycle tests. Upon further embedding in PDMS, a wearable pressure and strain sensing device has been prepared and shows excellent stretchability and mechanical durability upon 1000 cycles.

\section{RESULTS}

\section{Design for Au PCL networks}

Figure 1a shows a schematic diagram of the fabrication process for a soft and freestanding transparent electrode. A single-layer, microfibrous, polycaprolactone $(\mathrm{PCL})$ uniform network settled with a coiled fibrous frame is firstly fabricated by MEW. Then, a bimaterial-based electrode is obtained by depositing a thin gold layer on the PCL network using standard sputtering technique. To improve the transparency, the $\mathrm{PCL}$ network consisting of square grids $(200 \mu \mathrm{m}$ by $200 \mu \mathrm{m})$ of $15 \mu \mathrm{m}$ PCL fiber is deposited like a screen window, shown in Figures S1a,b. Coiled PCL fibers with a width of $200 \mu \mathrm{m}$ surrounding the single-layer network support it and achieve the freestanding characteristic of this electrode (Figures S1c, d). Our fabricated network electrode shows both a great transparency and an ultra-flexible performance (Movie S1 and Figure S4). In Fig. 1b, the logo of Aarhus University can be clearly seen through the folded $80 \mathrm{~nm}$ Au-coated sample, which illustrates the high transparency of our electrode. The SEM image shown in Fig. 1c exhibits a corresponding bended Au PCL network. Intuitively, there is no crack or any damage at microscale under bending. Both the smooth surface and great connection between the fibers provide effective paths for the electrons transporting through the networks without any barriers. The inset shows the corresponding EDS mapping image, which demonstrates that the upper bright side of the frame was coated with $\mathrm{Au}$ while the uncoated side presents dark. Contact angle for the front and back sides of obtained samples is displayed and quantified in Figure S2. The PCL substrate shows a typical water contact angle of $117^{\circ} .8$ After the deposition of $\mathrm{Au}, 20 \mathrm{~nm}$ Au-coated PCL network (20 Au PCL), $50 \mathrm{Au} \mathrm{PCL}$, and $80 \mathrm{Au} P C L$ samples are obtained. The Au-coated side displays a water contact angle of $113^{\circ}, 113^{\circ}, 114^{\circ}$, respectively, while the contact angle for the uncoated side of them is $105^{\circ}, 98^{\circ}, 96^{\circ}$, respectively (Figure S2,a, c). Flatter surfaces caused by the surface plasma treatment may result in a decrease in the contact angle on the uncoated side, which can be demonstrated in Figure S2,b.

\section{Optical and electric characteristic}

Figure $1 \mathrm{~d}$ shows plots of the sheet resistance versus transparency of the Au PCL networks with different thickness. For comparison, the performances of other structured materials from literatures are also shown. The $\mathrm{Au} P C L$ networks with a transmittance approaching $88 \%$ and $90 \%$ show a resistance of 1 and $2.8 \Omega$ $\mathrm{sq}^{-1}$, respectively, which present the best performance among Au-based electrodes. ${ }^{22,39,41,42}$ These values are only inferior to that of $\mathrm{Ag}$ nanowires, which is acceptable since bulk silver possesses better intrinsic electronic conductivity than bulk gold. ${ }^{43}$

The detailed transparency change along with the increasing thickness is presented in Figure S3. As expected, the transparency of our electrodes decreases slightly with the increasing of coating thickness (inset of Figure S3). These networks show a high transparency (>85\%) even with thicknesses $>80 \mathrm{~nm}$. It is worth noticing that there is only a slight decrease of the transparency for our oriented networks with the decreasing of sheet resistance, but it comes huge decrease for that of random nanowire or microwirebased electrodes. It could be attributed to the screen-window like array. ${ }^{44}$ The decreased resistance is generated from the increasing Au coating thickness on each fiber and does not affect the effective transmission paths of light, thus will hardly sacrifice the optical properties of the networks.

The effect of sheet resistance versus bending radius reflects the flexibility of the electrode, which is demonstrated in Figure S4. The Au PCL network-based electrode shows almost unchanged resistance when bending from the curvature radius of 100-1 mm, while the Au film-based electrode increased about $400 \%$ at the curvature radius of $1 \mathrm{~mm}$. Another characteristic of this electrode is its resistance variations under strain, as shown in Fig. 1e. Below 5\% stretching, the resistance remains basically unchanged, while it increases linearly from 6 to $20 \%$ strain.

\section{Conductive healing performances under stretching}

One of the most attractive characteristics for our Au PCL networkbased electrode is its micro-scale healability. The stretchingrelease-healing treatment is illustrated in Fig. 2a. After stretching, cracks are only formed at the Au coating layer, accompanied with an elongation for the beneath PCL network (Fig. 2b), confirming that the strain does not initiate any damage within the polymer network architecture. To heal the damaged Au layer, a heat treatment transferred via steam vapor is carried out to shrink the PCL fiber substrate by melting and relaxation, subsequently bringing the cracked Au coating layer regathered and contacted again, as shown in Fig. $2 \mathrm{~b}$ (from left to right). The corresponding detailed SEM and EDS images are presented in Figure S5. Compared with the original morphology of the fiber (Figure S5, a), there is a clear fracture on the Au coating after stretching (Figure S5,b); while after healing, the Figure S5,c confirms that the surface $A u$ coating layer is in contact again. The variations in representative resistances, $\left(R-R_{0}\right) / R_{0}$, are recorded at strain ranging from 0 to $50 \%$ (Fig. $2 \mathrm{c}$ ) for the samples with one cycle of stretching-release-healing, where the water vapor treatment was introduced for the healing process. The resistance increased up to 6 , and more than 1000 times after stretching to $20 \%$ and $50 \%$, respectively (labeled as "under stretching"). The resistance reduces to $600 \%$ after releasing from $20 \%$ strain, and to $3000 \%$ after releasing from $50 \%$ strain (data labeled as "after releasing"). Finally, the conductivity of them was observed to be recovered $70 \%$ and $25 \%$ to its initial value after vapor treatment, respectively (data labeled as "after healing without strain"). Figure $2 \mathrm{~d}$, e demonstrates the conductivity recovery of 10 cycles of stretchingrelease-healing tests under $5,10,20$, and $50 \%$ stretching.

Since PCL did not hydrolyze in a water solution after a period of 60 days at the temperatures approaching the polymer melting point of $60{ }^{\circ} \mathrm{C}^{45}$ and there is only less than $10 \mathrm{~s}$ contact in our healing process, we excluded the potential of the chemical effects of water vapor. During the stretching, the gold layer separates while the PCL fiber substrate elongates, inducing the increasing of resistance. Under vapor treatment, the PCL substrate exhibits a shrinking relaxation effect, forcing the gold layers to re-contact, as observed in Fig. 2 b. This great recovery ability is attributed to the 
a

\section{D-printing electrospun}

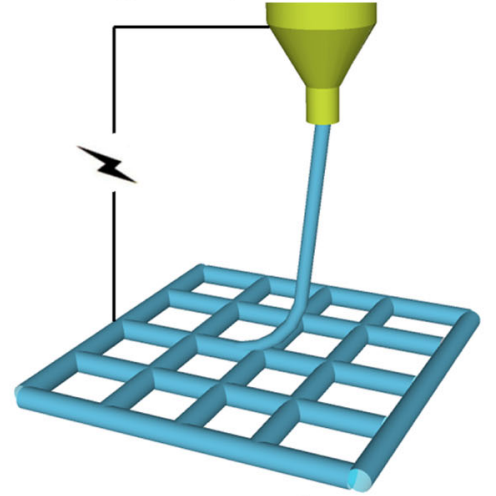

PCL networks

b

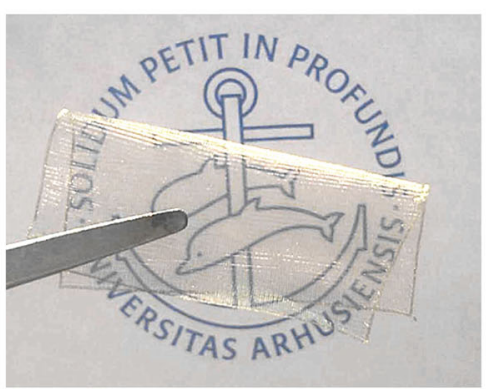

d

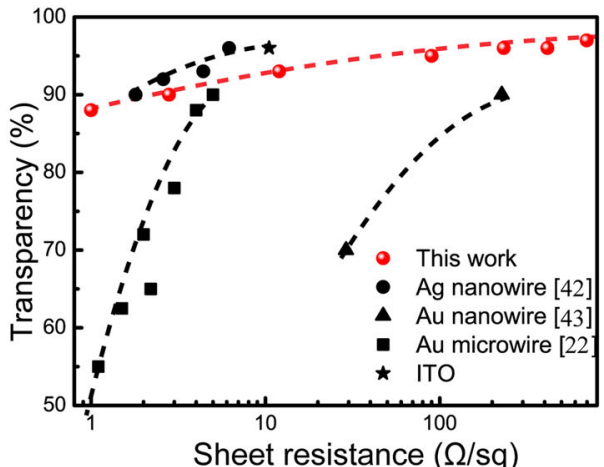

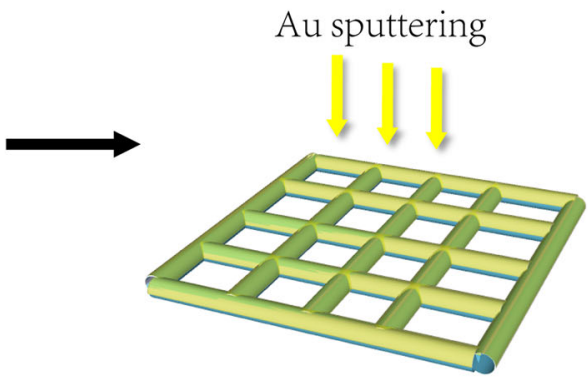

Au-coated PCL network

C

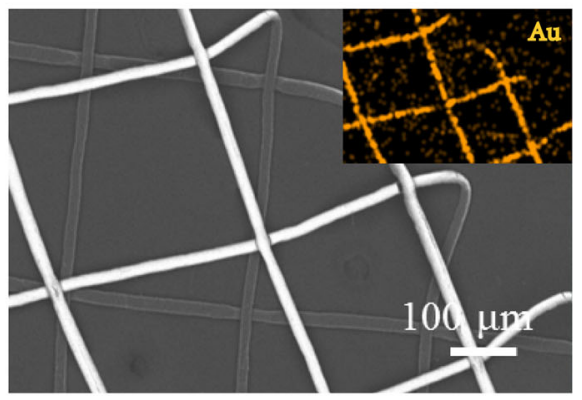

e

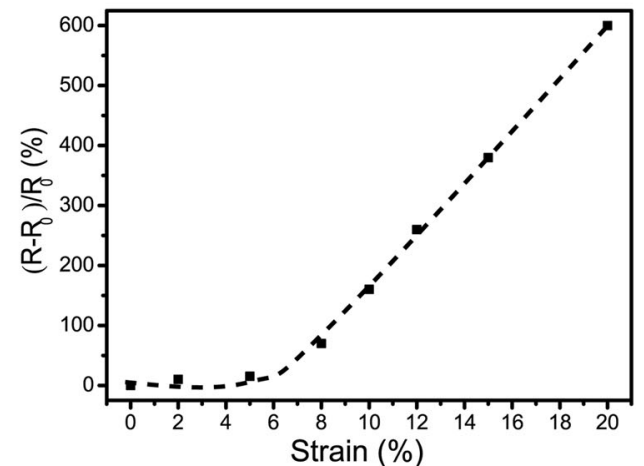

Fig. 1 a Schematic of Au network preparation method. b Photograph of an Au network electrode bended in half. Its transparency is illustrated by the visibility of the Aarhus university logo that is behind Au networks. c SEM images of the bended Au networks. Inset shows the corresponding EDS mapping image. d Sheet resistance versus optical transmission (at $550 \mathrm{~nm}$ ) for silver and Au networks. e Variations in relative resistance of $80 \mathrm{~nm}$-Au network at stretching strains ranging from 0 to $20 \%$

shrinking effect of PCL substrate, which could be further separated into two forces, one is adhesion force, another is capillary force. As we know, the dominating adhesion force in polymer has been proved to be the intermolecular van der Waals forces. ${ }^{33}$ The adhesion energy $(W)$ could reflect the strength of the interfacial van der Waals interactions, which can be expressed by the Eq. (1), ${ }^{46}$

$W=n * f * b *$

where $n^{*}$ is the attachments number per area and is proportional to mobility of the chain segments, $f^{*}$ is the typical van der Waals interactions, and $b^{*}$ is the necessary distance for breaking the interfacial interactions. Since $f^{*}$ and $b^{*}$ change slightly with temperature and are assumed to be constant, $W$ is determined by the mobility of chain segments and could be shown in an
Arrhenius relationship as follows, ${ }^{37}$

$$
W(T) \sim K \exp (-\Delta E / R T)
$$

where $K$ is a constant, $\Delta E$ is the activation energy, $R$ is the gas constant, and $T$ is the absolute temperature. Eq. (2) indicates an exponential increase of $W$ versus $T$. Moreover, molecular orientation of polymer chains decreases and crystallization decreases with increasing temperature, resulting in highly enhanced mobility of the chain on the fiber surface. ${ }^{33}$ Thus, there will be an abrupt increase of adhesion energy with the increasing temperature.

During the melt electrospinning process, flexible molten polymer chains at a fluid state are elongated along the axial direction by the high voltage. ${ }^{47}$ After being cooled, they are solidified and the mobility of them is restricted by the fiber 
a
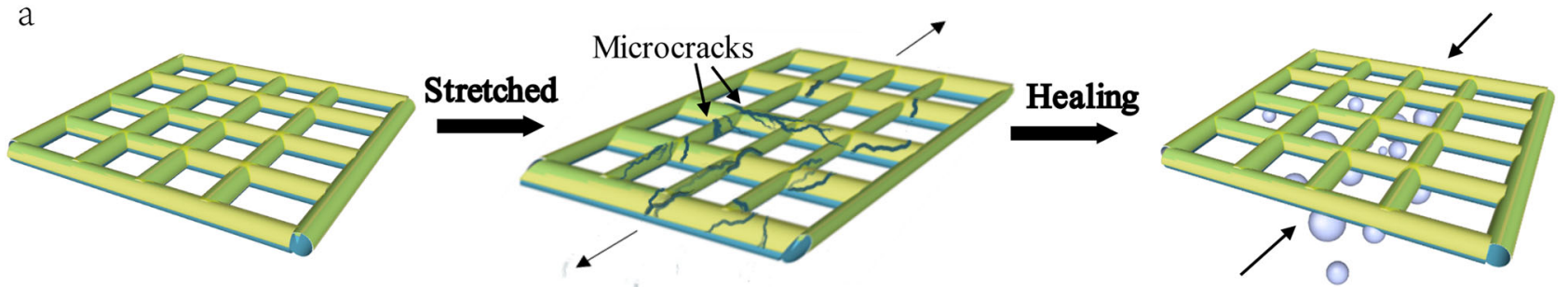

$\mathrm{b}$
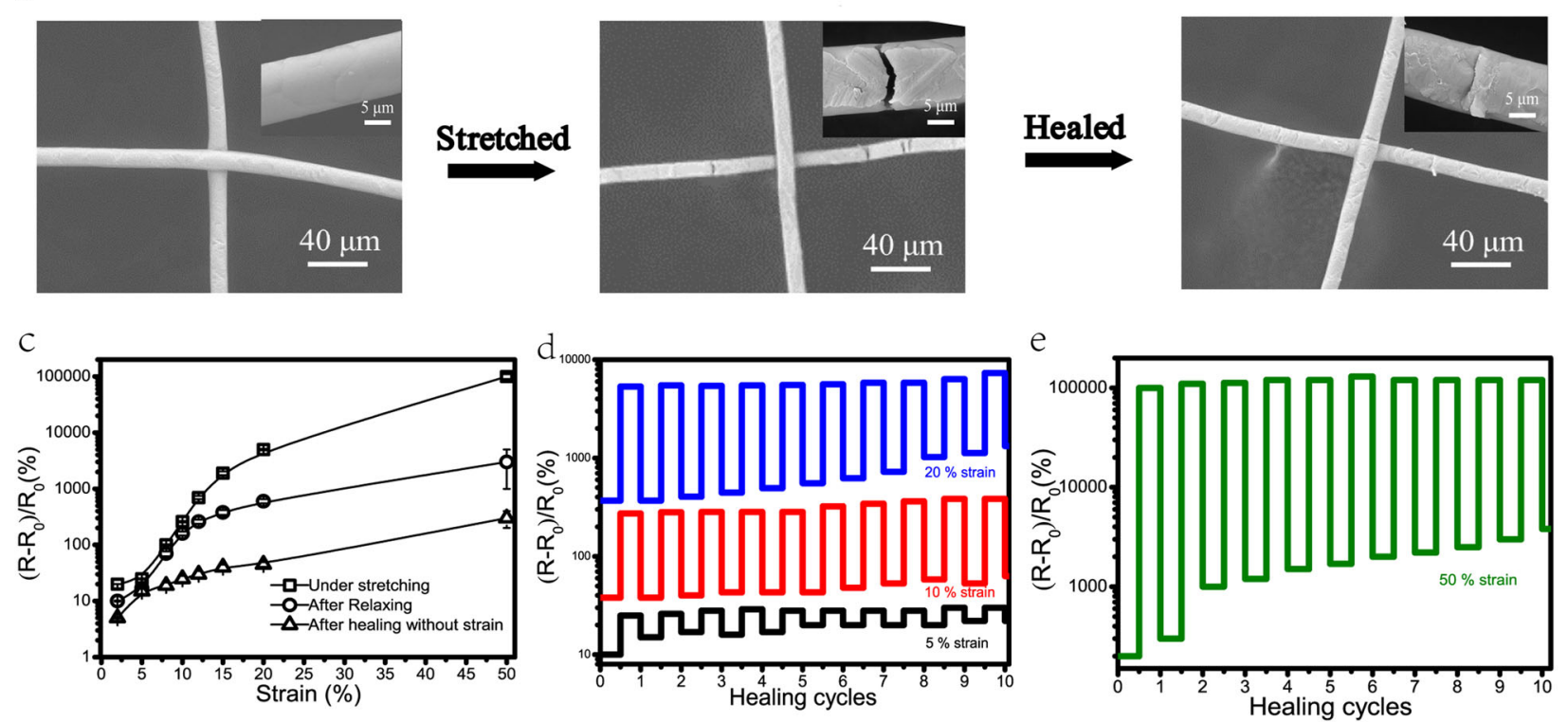

Fig. 2 a Representation of the treatments used for healing the Au network electrode. $\mathbf{b}$ SEM images for original, damaged and healed surface of Au microfiber (from left to right). c Change of the resistance of the Au network under stretching, after relaxing, and after healing without strain. d, e Resistance changes of the Au network for 10 stretching and healing cycles at 5, 10, 20, and 50\% strain

confinement effect. This confinement effect also exists when they are stretched or damaged at temperature below the melting point. As the steam vapor above the melting point is applied, the expanded polymer chains relax and shrink with the increasing adhesion energy, and moreover, the increasing chain mobility with the degree of crystallization and molecular orientation on the surface notably decreases, leading to a stress release. ${ }^{46,48}$ Moreover, water vapor condenses on PCL surface immediately, helping the network to cool down and avoids unnecessary welding and network damage.

It is worth noting that the Au layer remains in the solid state while the PCL polymer melt under steam, consequently there is a solid and liquid interface between the $A u$ layer and the $P C L$ substrate, which leading to a capillary bridge between them. ${ }^{49,50}$ Collectively, the Au film layer is proposed to suffer two strength force during vapor treatment, one is horizontal adhesion force $\left(F_{1}\right)$ and the other is vertical capillary force $\left(F_{2}\right)$. Figure 3a schematically illustrates the collective forces on the fracture area. Under the vapor treatment, the PCL fibers will be in a molten state, especially in the interfaces because metal exhibits better thermal conductivity. Thus, the Au-PCL interface is simultaneously subjected to contraction and capillary forces during healing. To prove such combined effect, the transition states during the healing process are observed. Based on the DSC result, the onset of melting and melting temperature of $\mathrm{PCL}$ networks are 40 and $60^{\circ} \mathrm{C}$, respectively. Therefore, morphology changes of $\mathrm{Au} P C L$ samples treated with vapor at those two temperatures are measured by SEM. As shown in Fig. 3b-g, PCL surface undergoes inwardly shrinking even without any previous strain, which indicates that the $\mathrm{Au}$ coating layer is not only subjected to a horizontal shrinkage force (adhesion force $F_{1}$ ) but also to vertical tension (capillary force $F_{2}$ ) under steam treatment. Thus, we proposed that the combination of van der Waals force and capillary force causes the injured surface to shrink like a wound to heal, and regaining the conductivity.

\section{Mechanical and stretching properties}

This healing performance of the Au PCL network electrode could be attributed to the great mechanical properties of micro-scale $\mathrm{PCL}$ fibers and the low melting temperature of PCL. The mechanical performance of Au-coated PCL networks is measured by uniaxial unconfined stretching in air at room temperature, as shown in Fig. 4a. The inset shows a photograph of $\mathrm{Au} P C L$ networks fixed in the mechanical measurement before stretching. To show the fiber diameter effect on mechanical properties, Fig. $4 \mathrm{~b}$ presents mechanical behavior of a single fiber with different diameters. A single, Au-coated PCL fiber with diameter of $150 \mu \mathrm{m}$ ruptured only after stretching to $30 \%$. However, when the size reduced by a factor of 10 , instead of broken, the fiber can maintain the plastic deformation to extend to $300 \%$, accompanied with a decrease in diameter (Figure S6). For our grid samples, a first failure occurs at about $30 \%$ strain for all samples, corresponding to the fracture of one side of the frame, made of rigid, welded, coiled fibers (Figure S1). While the second failure of another side of the frame happens at approximately $80 \%$ strain for uncoated PCL, 20 Au PCL and 50 Au PCL samples, and at $200 \%$ strain for $80 \mathrm{Au} \mathrm{PCL}$ (Fig. 4c). Attributed to great malleability of metal, thick Au coating layer improves the mechanical properties of the scaffold. The frame exhibit high Young's modulus, corresponding to 5, 9.1, 10.4, and $19.5 \mathrm{MPa}$ for PCL, $20 \mathrm{Au} \mathrm{PCL}, 50 \mathrm{Au} \mathrm{PCL}, 80 \mathrm{Au} \mathrm{PCL}$, 
a

Fracture $\quad 80 \mathrm{~nm}$ Au layer
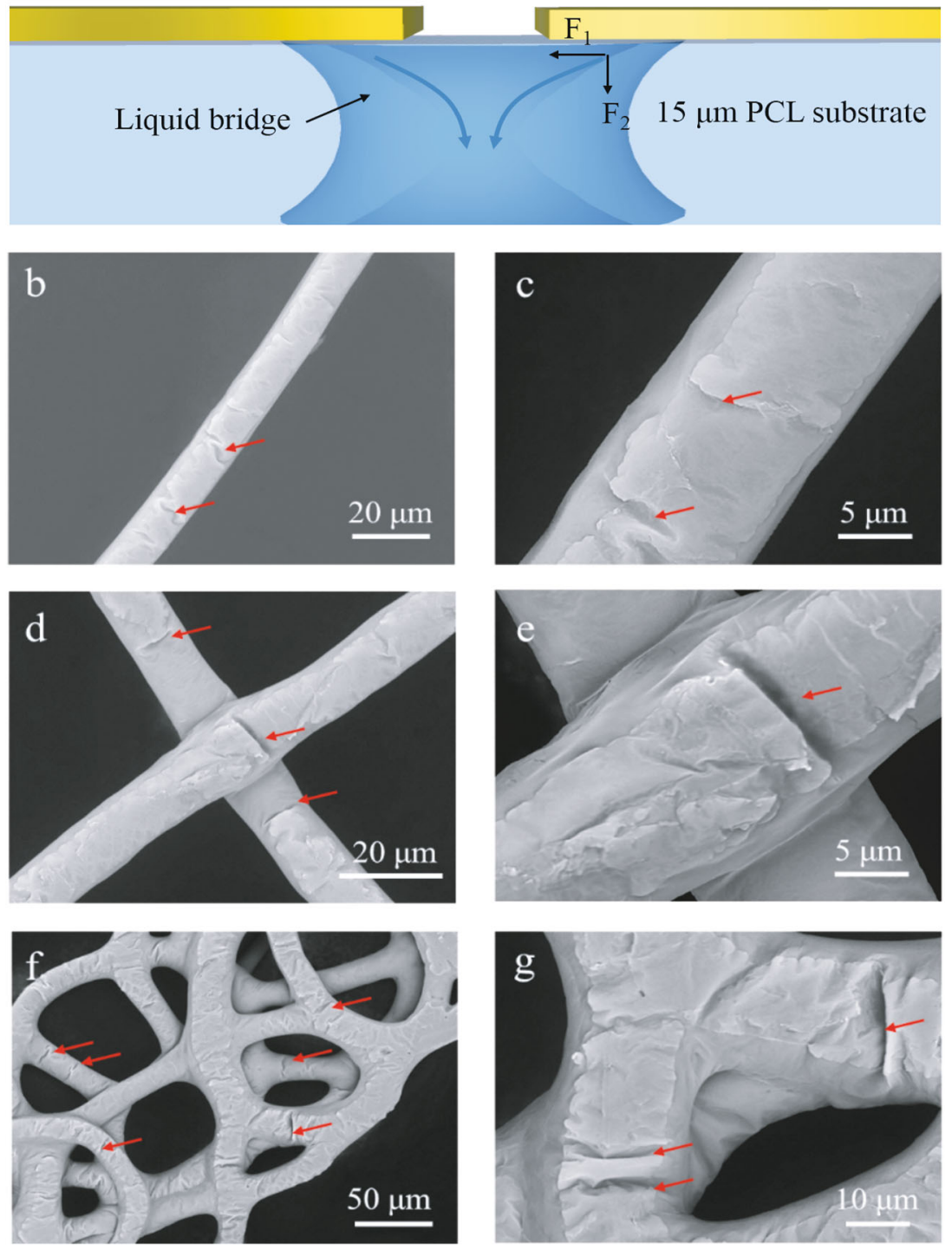

Fig. 3 Healing mechanism of van der Waals force combined with capillary force. a Schematic of adhesion force on the interface between Au and PCL layer connecting with a liquid bridge. $F_{1}$ represent the van der Waals force while $F_{2}$ represents the capillary force on the Au layer. $\mathbf{b}-\mathbf{g}$ The Au-coated PCL fiber after $40^{\circ} \mathrm{C} \mathbf{b}, \mathbf{c}$ and after $60^{\circ} \mathrm{C}$ steam treatments $\mathbf{d}-\mathbf{g}$. The shrinking positions are pointed out by red arrows

respectively (Fig. 4d). ${ }^{51}$ The grid part of this Au PCL network, consisted of straight, $15 \mu \mathrm{m}$ fibers, continues stretching without any fracture up to $330 \%$ strain. Consistent with the single fiber measurement, the great plasticity of $15 \mu \mathrm{m} \mathrm{PCL}$ allows our Au PCL network to maintain its original morphology during stretching, while all the damages of the Au coating exclusively appear on the surface of $\mathrm{PCL}$, and thereby facilitating the $\mathrm{Au}$ recontacting process by precisely healing at its original fracture site (see Fig. 2b; Figures S5,b, c).

\section{Cutting and welding performance}

Furthermore, the electrical healing capability is evaluated (Movie S2). For comparison, Movie S3 shows the process of an electrode cutting and connecting without healing. As shown in Fig. 5a, the ultra-thin and highly transparent electrode was connected to a circuit with a LED and a DC power supply. When cutting across the thin network with a scalpel, the circuit becomes an open circuit and the LED immediately goes off. The cut results in a breakage on the top gold layer as well as the bottom PCL fibers, signifying that the $\mathrm{Au} P C L$ networks incur severe damage. After welding with steam vapor at $70^{\circ} \mathrm{C}$ for $10 \mathrm{~s}$, the entire network electrode could be conductive again. SEM images in Fig. 5b exhibit an overlap of the broken networks, and the magnified image confirmed that these broken fibers are welded together. To confirm the broken network is healed, we also tested the tensile curve of the sample after a cutting and healing cycle. It is shown that the tensile strength of the coiled fibrous frame is reduced $37 \%$ (fracture at around $25-30 \%$ strain), while there is only $17 \%$ decrease for the straight fibers in the center, as shown in Figure S1,a, after water exposure (the tensile strength decreasing from $\sim 580$ to $480 \mathrm{KPa}$ ). It is noteworthy that the networks could be still extended to $300 \%$ without broken into two parts (see Figure S7). As shown in Fig. 5c, the resistance of $\mathrm{Au} P C L$ network across the cutting-healing process increases twice after the first cycle and about 50 times 


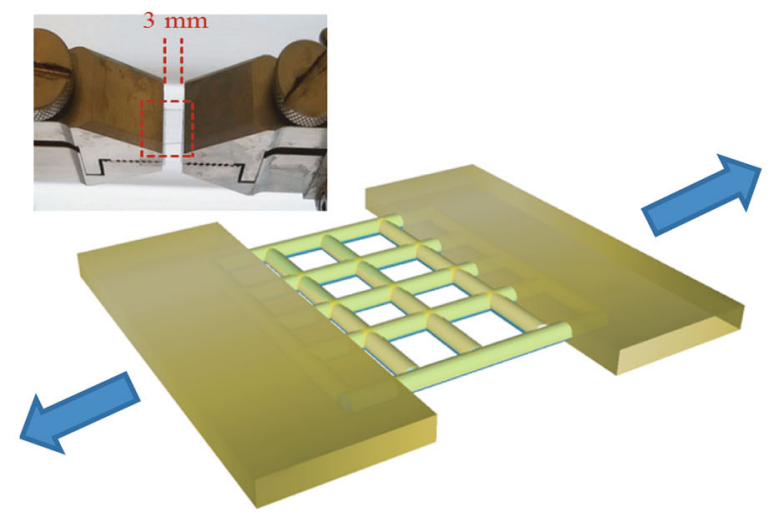

C

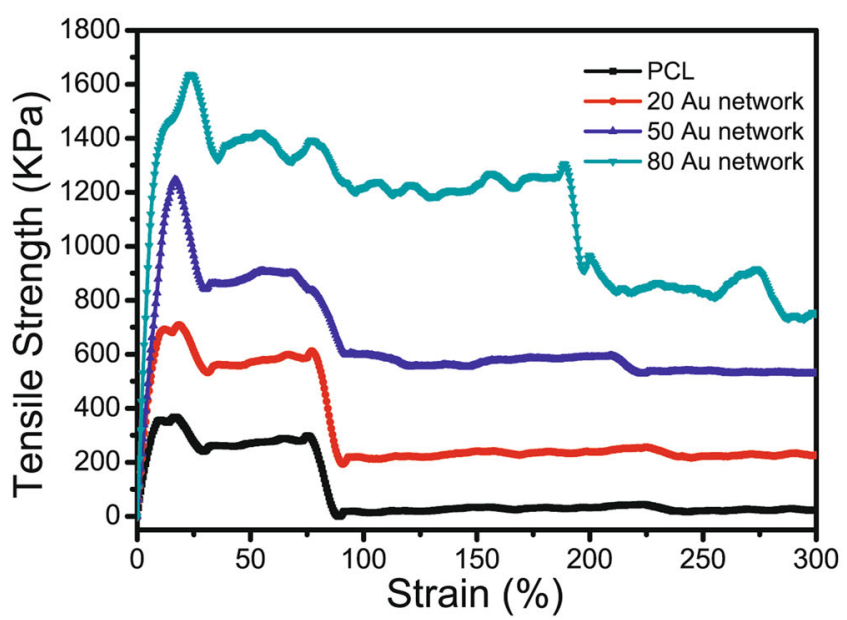

b

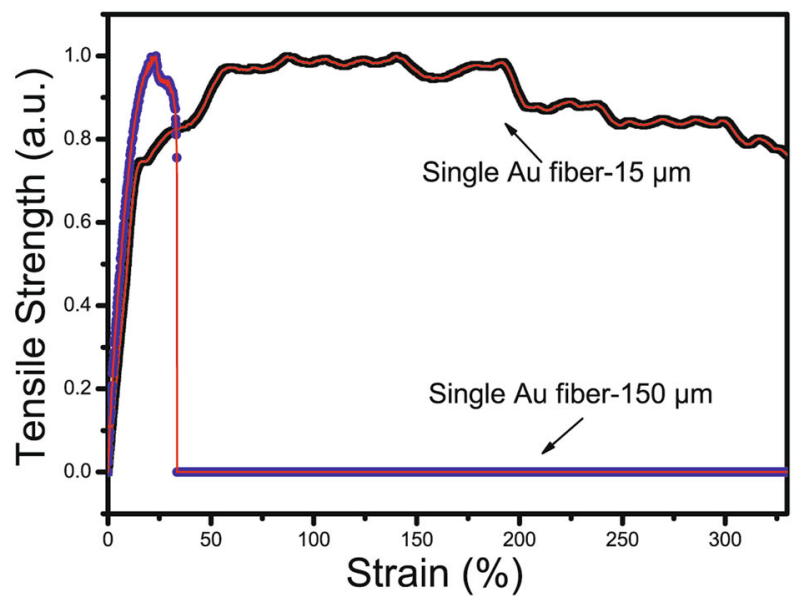

d

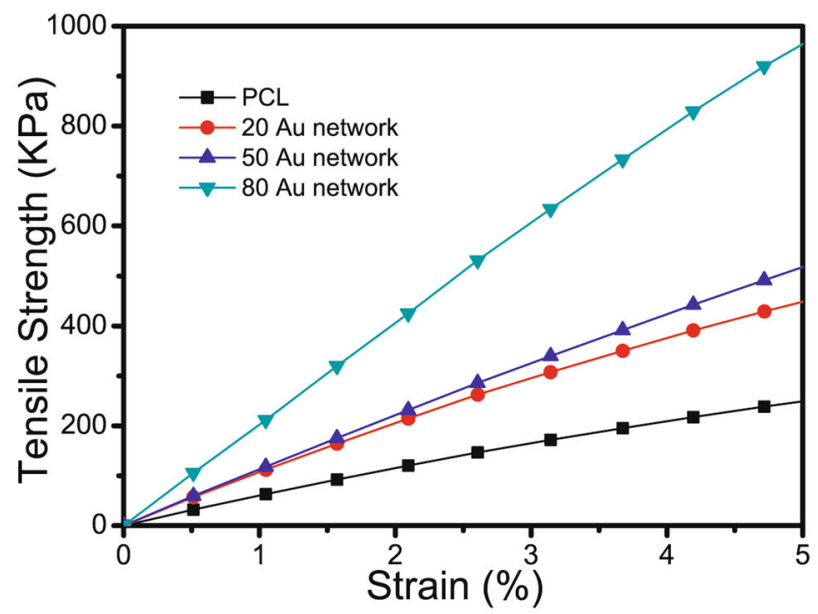

Fig. 4 a Schematic of the mechanical device. Inset shows a Photograph of Au networks before stretching. b Tensile strength single Au microfiber with different diameter of 15 or $150 \mu \mathrm{m}$. c, d The tensile stress-strain curves of PCL networks without coating and coated with 20, 50 , and $80 \mathrm{~nm}$ Au particles

after 10 cutting-healing cycles, presenting great promise of the micro-scale healing for repairing the damaged circuit.

\section{Assembled wearable pressure/strain sensor}

Finally, a stretchable, wearable pressure/strain sensor is assembled by comfining this healable electrode in Polydimethylsiloxane (PDMS). It has been reported such confinement effect can enhance the polymer chain dynamics of the amorphous regions and restrict growth of large crystallites. ${ }^{52}$ As a result, this effect can alter physical properties of polymers, such as lowering the mechanical modulus or increasing the mechanical ductility, which are all desirable for improving the stretchability. Figure 6 a shows a photograph of this confined Au PCL networks. Figure S8 shows the optical microscopy images for the structural change in the confined sensor under stretching. Under stretching, this sensor shows an elongation in length and a shrink in width due to Poisson's law, ${ }^{53}$ which is consistent with the result that the resistance increases linearly along with tensile strain. The surrounding PDMS evidently help to keep the morphology of the network as there is no fracture or buckling observed after releasing the strain. Furthermore, this flexible sensor can be simply attached to the skin of a human finger (inset of Fig. 6a). The pressure- and strain-sensitive properties of the prepared sensor are measured by an actuator, shown in Fig. $6 \mathrm{~b}$. We used the same uniaxial stretching mode as used for the mechanical test to measure this property. The normalized resistance change $R-R_{0} / R_{0}$ was measured, where $R$ and $R_{0}$ were the resistance for the sensor with or without strain, respectively. The gauge factor (GF) of a sensor is defined as GF $=\left(\Delta R / R_{0}\right) / \Delta \varepsilon$, where $\varepsilon$ is strain and $\Delta R / R_{0}$ is relative resistance change. Then, GF could be calculated by linear fitting, and the value is $5.3 \pm 0.2$ (in the strain of $0-50 \%$ ). Meanwhile, the relating pressure versus. resistance plot is shown in Fig. $6 \mathrm{~d}$, which exhibits a good linear relationship in the ranging from 0 to $30 \mathrm{KPa}$. This good linear relationship between the resistance and pressure for this $\mathrm{Au} P C L$ network suggests its potential application as both strain and pressure sensor. During repeated 1000 cycles, this sensor exhibits great stable and reversible stretchability under 50\% stretching, as shown in Fig. 6 e. Figure $6 f$ compared the resistance changes between the Au $\mathrm{PCL}$ network confined in PDMS and the unconfined $\mathrm{Au} P C L$ network under strain cycling. In the beginning, it would increase $40 \%$ in the first 50 cycles, due to relaxation from the confinement, and keep stable elastic character in the following cycles (Figure S9). ${ }^{54}$ The resistance variation is between 0 and $100 \%$ during cycling for confined Au PCL network while it increases more than $10^{6} \%$ for the unconfined one after only one cycle. Thus, our confined Au PCL network shows great stability, indicating its suitable application of a pressure-sensing device. 
a
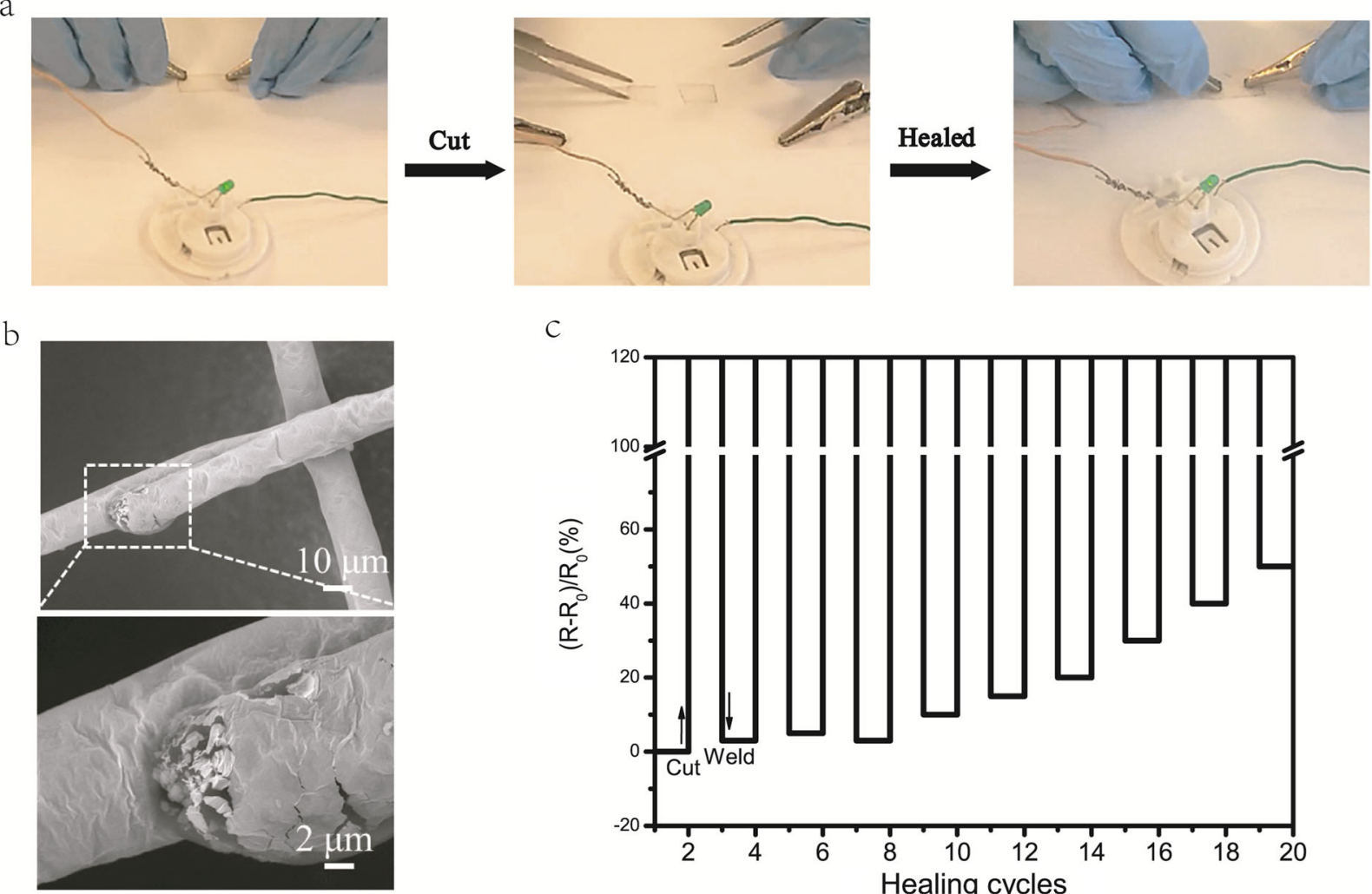

C

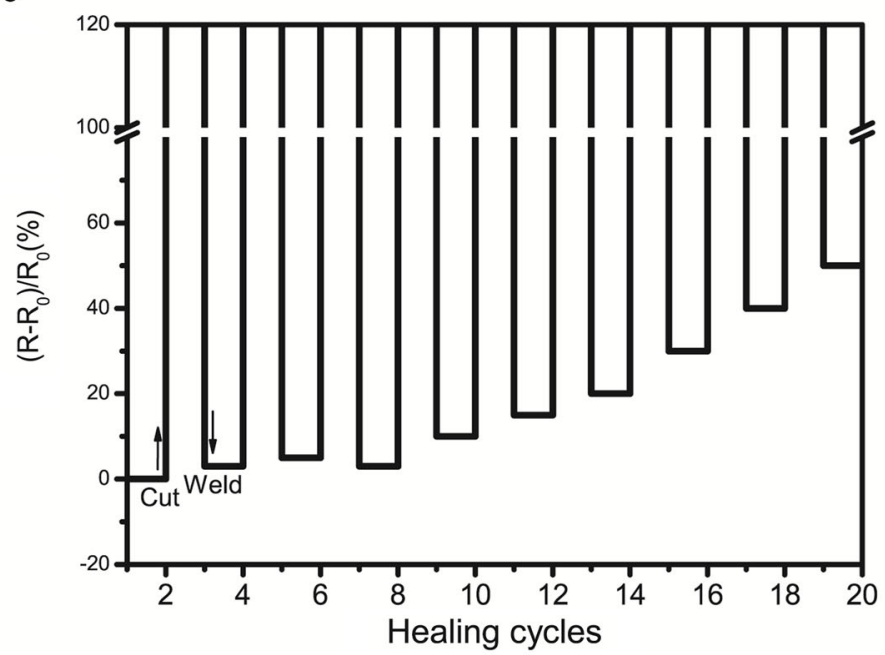

Fig. 5 a Images of Au network-based transparent electrode connected with a LED light in a circuit. From left to right is the original, after damaged, and after healing state of this network. The entire welding process is shown in Supporting video 2. b Morphology of an Au network after welding. c Resistance changes of the Au network during 10 cut and healing cycles

\section{DISCUSSION}

Here, highly conductive, transparent, and ultra-flexible fiber-based biomaterial electrodes are prepared by melt electrospinning followed by Au coating. More importantly, a water vapor method was successfully utilized to achieve a micro-scale healing of fiberbased electrodes. By rapid healing at their original micro-scale fracture sites, this approach can quickly accomplish recovering of its resistance after a tensile failure under $5,10,20,50 \%$ strain. Even under $50 \%$ stretching, the resistance of the networks increases more than a thousand times, it will be reduced by a factor of 10 after this facile vapor treatment. And this performance exhibits stable and repeatable under 10 cycling tests. The regaining of the conductivity could be contributed to the energy transfer of water vapor through both van der Waals force and capillary force at this bimaterial interface. Finally, a wearable pressure/strain sensor is fabricated by confining the networks inside a PDMS substrate. Based on the confinement effect, this pressure/strain sensor displays a good linear relationship between the resistance and pressure/strain, and such sensibility can be maintained under $50 \%$ stretching upon 1000 cycles.

\section{METHODS}

Electrospinning combined with 3D printing

PCL polymer $\left(M_{n}=45000\right)$ was purchased from Sigma-Aldrich and used as received. The controlled electrospinning was carried out by a melt electrospinning instrument (CAT000111, Spraybase, Ireland). PCL pellets were first loaded into a stainless-steel syringe. The syringe was protected by placing in a custom plexiglass housing and heated by an electronic control heater. After heating the syringe to $70^{\circ} \mathrm{C}$ for one hour, a homogeneous melt polymer was obtained, as the DSC test shows bulk PCL melt around $70^{\circ} \mathrm{C}$ (Figure S10). A stainless-steel needle (24 G) was attached to the syringe and used as the spinneret. The melted PCL was injected into the needle with a flow rate controlled by pressure of $0.25 \mathrm{bar}$ using a Spraybase ${ }^{\circledR}$ Pressure Driven System. A $12 \mathrm{~cm} \times 9 \mathrm{~cm}$ thin aluminum plate was used as a collector, and a high voltage of $3.5 \mathrm{kV}$ was applied to the plate while the needle was connected to the ground $(4 \mathrm{~mm}$ distance between them). There are two linear slide assemblies in an $x-y$ configuration mounted onto the collecting plate to control stage movement with a translation velocity of $2000 \mathrm{~mm} \mathrm{~min}^{-1}$. Custom translation patterns were realized by editing G-code using the Mach3 motion control software. The printed freestanding $P C L$ networks were subsequently coated with gold with different thickness $(10,20,30,40,50$, 60 , and $80 \mathrm{~nm}$ ) by a sputter coater (EM SCD500, Leica, Germany). For comparison, Au films were also prepared by Au sputtering on polyethylene terephthalate (PET) substrates with the same conditions. As a polymer matrix for stretchable sensors, PDMS film was prepared by adding the curing agent into PDMS solution at a concentration of $8 \%$ and uniformly mixing (Sylgard 184, Dow Corning, USA). The liquid mixture was then transferred into a vacuum oven to remove the air and cured at $60^{\circ} \mathrm{C}$ until it was completely solidified.

\section{Characterizations}

The optical transmission spectrum was measured by a Multilabel Reader (PerkinElmer 2030, USA). The transparency is defined as the optical transmission (at $550 \mathrm{~nm}$ ), which were measured for Au-coated PCL networks with different thickness $(10,20,30,40,50,60$, and $80 \mathrm{~nm})$. The networks are fixed on the surface of a transparent plastic substrate. The plastic substrate was set as baseline. A four-probe measurement was used to obtain sheet resistances to avoid the contact resistance and every reported value is the average value of at least five measurements (34450A Digital Multimeter, Keysight Technologies, USA). A square contact geometry with a distance of $3 \mathrm{~mm}$ between each probe was used for the measurement. The Au films were prepared on PET for bending tests while Au PCL network electrodes were presented without PET substrate for both bending and tensile tests. To assemble and test the pressure/strain device, a $2 \times 2 \mathrm{~cm}^{2}$ Au PCL network with sheet resistance of $2.8 \Omega \mathrm{sq}^{-1}$ was embedded in a PDMS matrix. For comparison, an Au PCL network with the same sheet resistance was also prepared. The mechanical performance of 
a

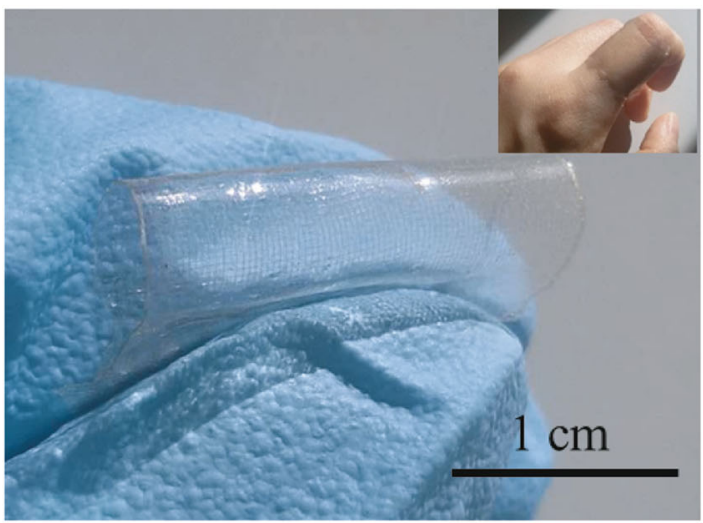

C

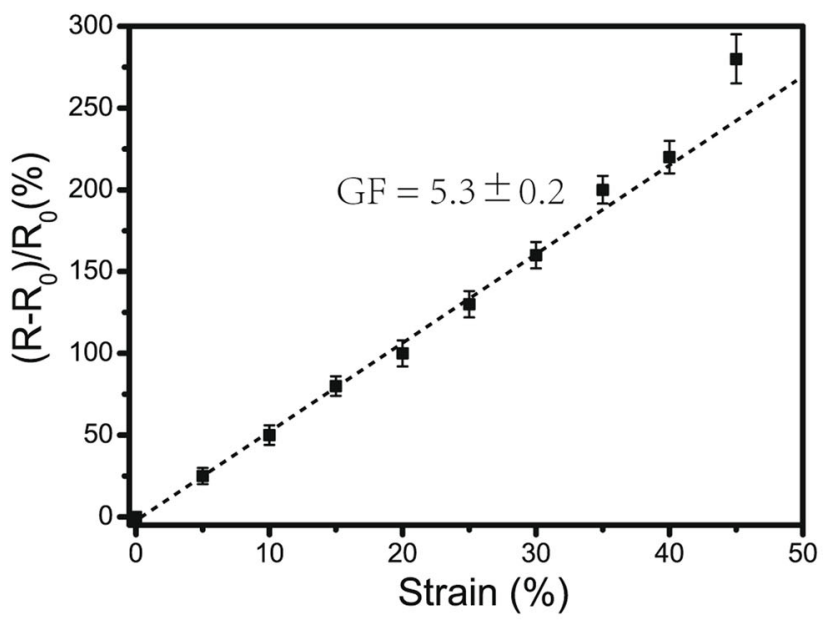

e

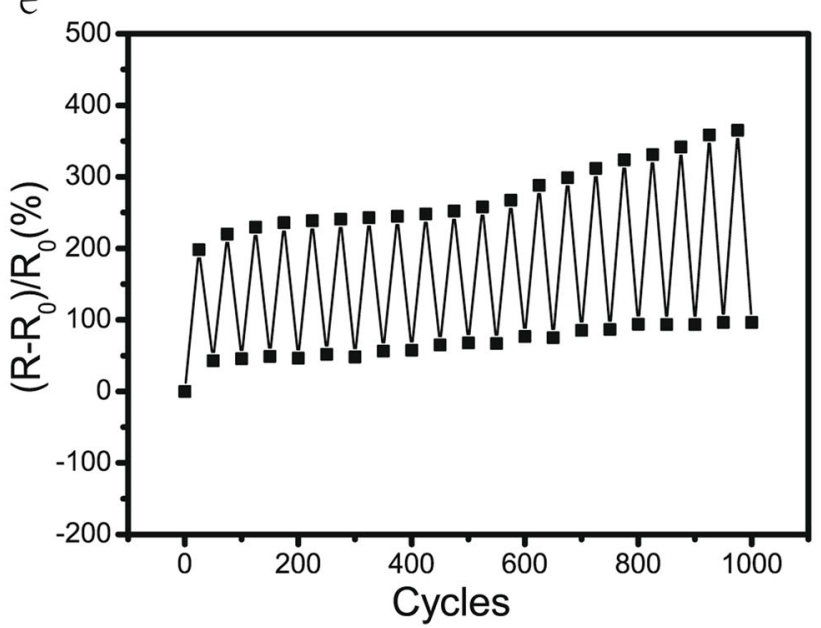

b

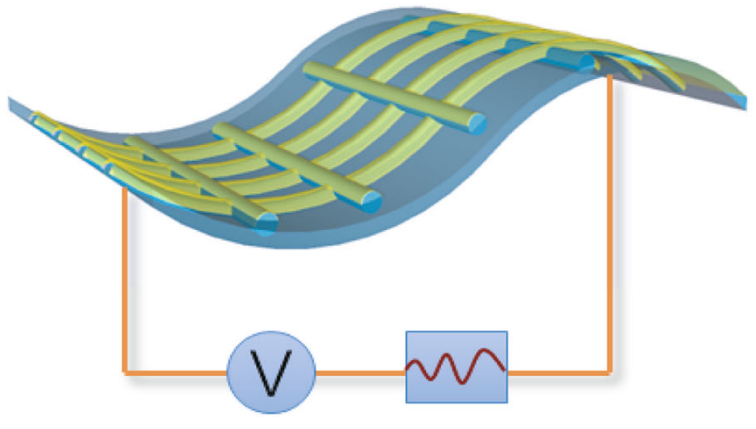

d

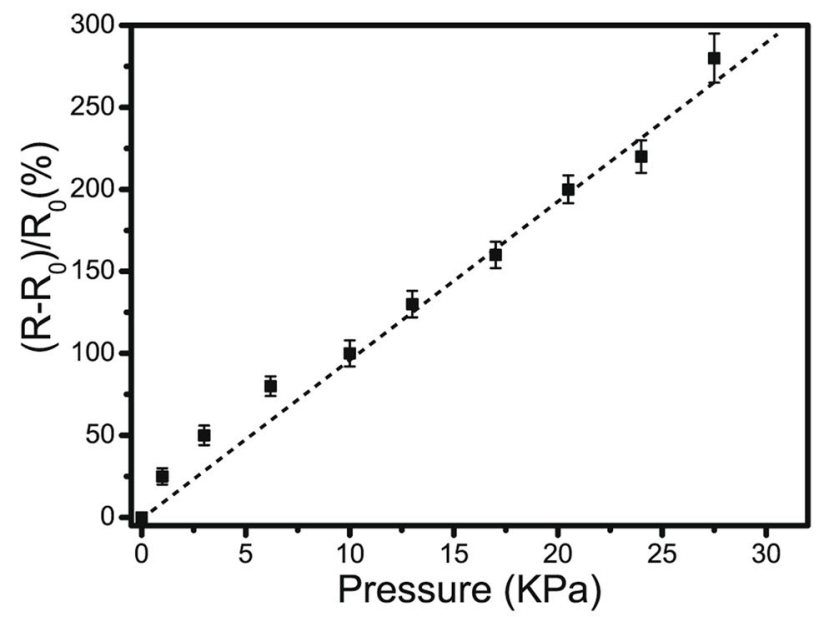

f

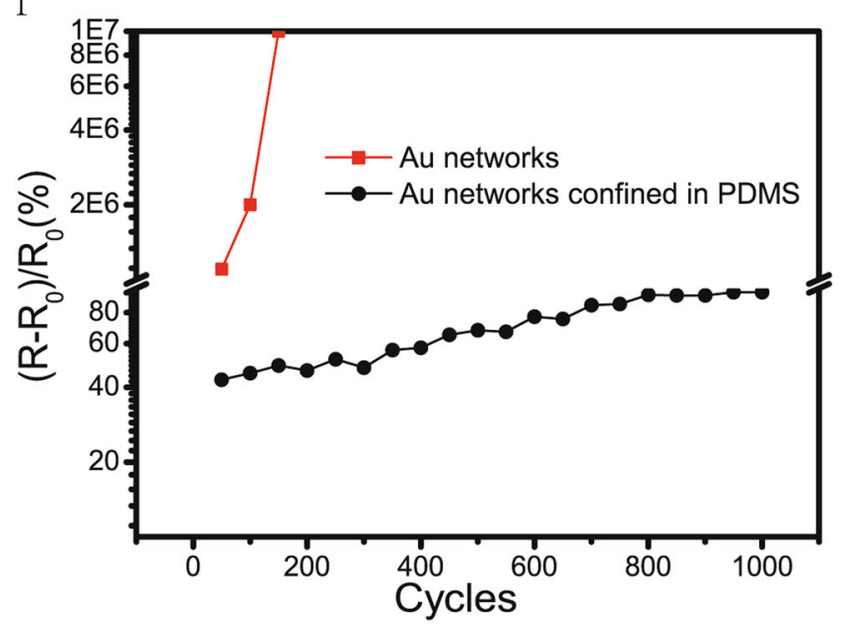

Fig. 6 a Photograph of embedded Au networks with thickness of $80 \mathrm{~nm}$. Inset shows that the flexible sensor attached to a finger. $\mathbf{b}$ Illustration diagram of Au networks embedded in PDMS matrix and connected in a circuit. c, d Resistance change in $\Delta R / R_{0}$ of Au network sensor at stretching strains ranging from 0 to $50 \%$ or pressure ranging from 0 to $30 \mathrm{KPa}$. e Variations in relative resistance during 1000 stretching cycles at $50 \%$ strain. f Comparison of resistance changes between the Au network confined in PDMS and the unconfined Au network under strain cycling

Au-coated PCL networks was measured by an ElectroForce test instrument with a strain rate of $0.1 \mathrm{mms}^{-1}$ (3200 Series II, Bose, USA). The electromechanical properties were carried out by a combination of the ElectroForce test instrument connecting to a multimeter (973A, HewlettPackard, USA). Optical microscopy images of Au PCL networks embedded in PDMS under stretching and releasing were taken with an inverted optical microscope (EVOS FL Auto, Thermo Fisher Scientific, USA).
Morphologies of $\mathrm{Au} \mathrm{PCL}$ networks were characterized by a scanning electron microscope (Hitachi, TM3030). Differential scanning calorimetry (DSC) was conducted by a Pyris 6 DSC system (Perkin-Elmer Cetus, Norwalk, USA). Approximately $8 \mathrm{mg}$ of the sample was added to an aluminum crucible and measured under nitrogen at a rate of $20 \mathrm{~mL} \mathrm{~min}{ }^{-1}$. An empty crucible was supplied as an inert reference. The thermal was 
applied with a heating scan from 20 to $100^{\circ} \mathrm{C}$ at a heating rate of $10^{\circ} \mathrm{C}$ $\mathrm{min}^{-1}$.

\section{Healing process}

For the controlled healing treatment, the damaged $\mathrm{Au} P C L$ networks were exposed to steam vapor for $10 \mathrm{~s}$ at $70^{\circ} \mathrm{C}$, since DSC measurement indicates that PCL networks show a melting temperature at $59.7^{\circ} \mathrm{C}$ (Figure S10). The steam was provided by heating the deionized water in an opened jar, and the temperature of the opening area was determined by an electronic thermometer. When the vapor contacted with the networks, it released heat and liquefied into water droplets that evenly and completely repaired the network. It is also worth mentioning that the coated gold layer improves this process since metal can conduct heat more efficiently.

\section{DATA AVAILABILITY}

The datasets generated and analyzed during the current study are available from the corresponding authors on reasonable request.

\section{ACKNOWLEDGEMENTS}

This research was supported by grants from the Danish National Research Foundation (grant no. DFF-6108-00396), Young Investigator Program from the Villum Foundation (grant no. VKR022954), AUFF NOVA-Project (grant no. AUFF-E2015-FLS-9-18), EU H2020 (MNR4SCELL no. 734174) and International Technological Collaboration Project of Shanghai (grant no. 17520710300), National Natural Science Foundation of China (grant no. 51671136) and Research start-up funds of DGUT (grant no. GC300501-17). The authors thank the helpful discussions with Sampson Anankanbil.

\section{AUTHOR CONTRIBUTIONS}

Y.W. and Y.S. designed the processes and experiments. Y.W., Y.S., Z.W., and Z.Z. fabricated and characterized the materials. M.D., L.C., and M.C. supervised the overall research. Y.W., Y.S., M.D., M.C., and X.H. contributed to the writing of the manuscript.

\section{ADDITIONAL INFORMATION}

Supplementary information accompanies the paper on the npj Flexible Electronics website (https://doi.org/10.1038/s41528-018-0043-z).

Competing interests: The authors declare no competing interests.

Publisher's note: Springer Nature remains neutral with regard to jurisdictional claims in published maps and institutional affiliations.

\section{REFERENCES}

1. Someya, T., Bao, Z. \& Malliaras, G. G. The rise of plastic bioelectronics. Nature 540, 379-385 (2016)

2. Bradley, D. D. C. \& Huang, W. A new flexible venue. npj Flex. Electron. 1, 3 (2017).

3. Wang, B., Bao, S., Vinnikova, S., Ghanta, P. \& Wang, S. Buckling analysis in stretchable electronics. npj Flex. Electron. 1, 5 (2017).

4. Zhao, J. et al. Recent developments of truly stretchable thin film electronic and optoelectronic devices. Nanoscale 10, 5764-5792 (2018).

5. Li, Y., Chen, S., Wu, M. \& Sun, J. Polyelectrolyte multilayers impart healability to highly electrically conductive films. Adv. Mater. 24, 4578-4582 (2012).

6. Gong, C. et al. A healable, semitransparent silver nanowire-polymer composite conductor. Adv. Mater. 25, 4186-4191 (2013).

7. Zhang, Q., Liu, L., Pan, C. \& Li, D. Review of recent achievements in self-healing conductive materials and their applications. J. Mater. Sci. 53, 27-46 (2018).

8. Wang, Y. et al. Low-cost, $\mu$ m-thick, tape-free electronic tattoo sensors with minimized motion and sweat artifacts. npj Flex. Electron. 2, 6 (2018).

9. Gupta, S., Navaraj, W. T., Lorenzelli, L. \& Dahiya, R. Ultra-thin chips for highperformance flexible electronics. npj Flex. Electron. 2, 8 (2018).

10. Bader, C., Kolb, D., Weaver, J. C. \& Oxman, N. Data-driven material modeling with functional advection for 3D printing of materially heterogeneous objects. $3 D$ Print. Addit. Manuf. 3, 71-79 (2016).

11. Keating, S. J., Leland, J. C., Cai, L. \& Oxman, N. Toward site-specific and selfsufficient robotic fabrication on architectural scales. Sci. Robot. 2, eaam8986 (2017).
12. Zhu, J., Dexheimer, M. \& Cheng, H. Reconfigurable systems for multifunctional electronics. npj Flex. Electron. 1, 8 (2017).

13. Zhang, S. \& Cicoira, F. Water-enabled healing of conducting polymer films. Adv. Mater. 29, 1-6 (2017).

14. Darabi, M. A. et al. Correction to: skin-inspired multifunctional autonomic-intrinsic conductive self-healing hydrogels with pressure sensitivity, stretchability, and 3D printability (Advanced Materials, (2017), 29, 31, (1700533), 10.1002/ adma.201700533). Adv. Mater. 30, 1-8 (2018).

15. Han, L. et al. A mussel-inspired conductive, self-adhesive, and self-healable tough hydrogel as cell stimulators and implantable bioelectronics. Small 13, 1-9 (2017).

16. Kang, J. et al. Tough and water-insensitive self-healing elastomer for robust electronic skin. Adv. Mater. 1706846, 1-8 (2018).

17. Yu, K. J., Yan, Z., Han, M. \& Rogers, J. A. Inorganic semiconducting materials for flexible and stretchable electronics. npj Flex. Electron. 1, 4 (2017).

18. Zou, Z. et al. Rehealable, fully recyclable, and malleable electronic skin enabled by dynamic covalent thermoset nanocomposite. Sci. Adv. 4, 1-9 (2018).

19. Lee, S. et al. A transparent bending-insensitive pressure sensor. Nat. Nanotechnol. 11, 472-478 (2016)

20. Yamada, T. et al. A stretchable carbon nanotube strain sensor for human-motion detection. Nat. Nanotechnol. 6, 296-301 (2011).

21. Tiwari, N. et al. Healable and flexible transparent heaters. Nanoscale 9 14990-14997 (2017).

22. Yang, J., Bao, C., Zhu, K., Yu, T. \& Xu, Q. High-performance transparent conducting metal network electrodes for perovksite photodetectors. ACS Appl. Mater. Interfaces 10, 1996-2003 (2018).

23. Lee, J. H. et al. Cu microbelt network embedded in colorless polyimide substrate: flexible heater platform with high optical transparency and superior mechanical stability. ACS Appl. Mater. Interfaces 9, 39650-39656 (2017).

24. Bai, X. et al. Room-temperature processing of silver submicron fiber mesh for flexible electronics. npj Flex. Electron. 2, 3 (2018).

25. Han, S. et al. Fast plasmonic laser nanowelding for a Cu-nanowire percolation network for flexible transparent conductors and stretchable electronics. Adv. Mater. 26, 5808-5814 (2014).

26. Garnett, E. C. et al. Self-limited plasmonic welding of silver nanowire junctions. Nat. Mater. 11, 241-249 (2012).

27. Tan, Y. J., Wu, J., Li, H. \& Tee, B. C. K. Self-healing electronic materials for a smart and sustainable future. ACS Appl. Mater. Interfaces 10, 15331-15345 (2018).

28. Li, Y., Chen, S., Wu, M. \& Sun, J. Rapid and efficient multiple healing of flexible conductive films by near-infrared light irradiation. ACS Appl. Mater. Interfaces 6, 16409-16415 (2014).

29. Parida, K. et al. Highly transparent, stretchable, and self-healing ionic-skin triboelectric nanogenerators for energy harvesting and touch applications. Adv. Mater. 29, 1-8 (2017).

30. Adly, N. et al. Printed microelectrode arrays on soft materials: from PDMS to hydrogels. npj Flex. Electron 2, 1-9 (2018).

31. Nassar, J. M. et al. Compliant lightweight non-invasive standalone 'Marine Skin' tagging system. npj Flex. Electron. 2, 13 (2018).

32. Palleau, E., Reece, S., Desai, S. C., Smith, M. E. \& Dickey, M. D. Self-healing stretchable wires for reconfigurable circuit wiring and 3D microfluidics. Adv. Mater. 25, 1589-1592 (2013).

33. Maeda, N., Chen, N., Tirrell, M. \& Israelachvili, J. N. Adhesion and friction mechanisms of polymer-on-polymer surfaces. Science. 297, 379-382 (2002).

34. Autumn, K. et al. Adhesive force of a single gecko foot-hair. Nature 405, 681-685 (2000).

35. Ambrosetti, A., Ferri, N., DiStasio, R. A. \& Tkatchenko, A. Wavelike charge density fluctuations and van der Waals interactions at the nanoscale. Science. 351, 1171-1176 (2016).

36. Xue, L., Kovalev, A., Eichler-Volf, A., Steinhart, M. \& Gorb, S. N. Humidity-enhanced wet adhesion on insect-inspired fibrillar adhesive pads. Nat. Commun. 6, 1-9 (2015).

37. Yang, Z., Fujii, Y., Lee, F. K., Lam, C.-H. \& Tsui, O. K. C. Glass transition dynamics and surface layer mobility in unentangled polystyrene films. Science. 328, 1676-1679 (2010).

38. Sun, B. et al. Recent advances in flexible and stretchable electronic devices via electrospinning. J. Mater. Chem. C. 2, 1209-1219 (2014).

39. $\mathrm{Wu}, \mathrm{H}$. et al. A transparent electrode based on a metal nanotrough network. Nat. Nanotechnol. 8, 421-425 (2013).

40. Miyamoto, A. et al. Inflammation-free, gas-permeable, lightweight, stretchable on-skin electronics with nanomeshes. Nat. Nanotechnol. 12, 907-913 (2017)

41. Huang, Y. et al. Large-scale spinning of silver nanofibers as flexible and reliable conductors. Nano. Lett. 16, 5846-5851 (2016).

42. Maurer, J. H. M., González-García, L., Reiser, B., Kanelidis, I. \& Kraus, T. Templated self-assembly of ultrathin gold nanowires by nanoimprinting for transparent flexible electronics. Nano. Lett. 16, 2921-2925 (2016). 
43. Lide, D. R. Handbook of Chemistry and Physics. 81st edn (CRC Press, Boca Raton, 2000).

44. Hu, L., Wu, H. \& Cui, Y. Metal nanogrids, nanowires, and nanofibers for transparent electrodes. Mrs. Bull. 36, 760-765 (2011).

45. Siparsky, G. L., Voorhees, K. J. \& Miao, F. Hydrolysis of polylactic acid (PLA) and polycaprolactone $(\mathrm{PCL})$ in aqueous acetonitrile solutions: autocatalysis. J. Environ. Polym. Degrad. 6, 31-41 (1998).

46. Shi, Q. et al. Mechanism of adhesion between polymer fibers at nanoscale contacts. Langmuir 28, 4663-4671 (2012).

47. Reneker, D. H. \& Chun, L. Nanometre diameters of polymer, produced by electrospinning. Nanotechnology 7, 216-223 (1996).

48. Shi, Q., Wan, K. T., Wong, S. C., Chen, P. \& Blackledge, T. A. Do electrospun polymer fibers stick? Langmuir 26, 14188-14193 (2010).

49. Nikoobakht, B., Wang, Z. L. \& El-Sayed, M. A. Self-assembly of gold nanorods. J. Phys. Chem. B 104, 8635-8640 (2000).

50. Liu, Y. et al. Capillary-force-induced cold welding in silver-nanowire-based flexible transparent electrodes. Nano. Lett. 17, 1090-1096 (2017).

51. Croisier, F. et al. Mechanical testing of electrospun PCL fibers. Acta Biomater. 8, 218-224 (2012).

52. $\mathrm{Xu}, \mathrm{J}$. et al. Highly stretchable polymer semiconductor films through the nanoconfinement effect. Science 355, aah4496 (2017).
53. Liang, J. et al. Silver nanowire percolation network soldered with graphene oxide at room temperature and its application for fully stretchable polymer lightemitting diodes. ACS Nano 8, 1590-1600 (2014).

54. Liu, Z. et al. Surface strain redistribution on structured microfibers to enhance sensitivity of fiber-shaped stretchable strain sensors. Adv. Mater. 30, 1-8 (2018).

(i) Open Access This article is licensed under a Creative Commons Attribution 4.0 International License, which permits use, sharing, adaptation, distribution and reproduction in any medium or format, as long as you give appropriate credit to the original author(s) and the source, provide a link to the Creative Commons license, and indicate if changes were made. The images or other third party material in this article are included in the article's Creative Commons license, unless indicated otherwise in a credit line to the material. If material is not included in the article's Creative Commons license and your intended use is not permitted by statutory regulation or exceeds the permitted use, you will need to obtain permission directly from the copyright holder. To view a copy of this license, visit http://creativecommons. org/licenses/by/4.0/.

(c) The Author(s) 2018 